Gynaecological Oncology surgery between high/high and medium/low HDI country settings

Result(s)* GO SOAR1 is open to recruitment internationally. Conclusion* The GO SOAR Collaborative aims to improve surgical outcomes through collaborative research. It will provide risk adjusted patient level outcome data collected via a centralised database to advise HDI country group specific policy formation.

\section{NON-SURGICAL ASPECTS OF MINIMISING INTRAOPERATIVE HAEMORRHAGE IN COMPLEX PELVIC SURGERY - THE MULTIDISCIPLINARY APPROACH}

${ }^{1} \mathrm{C}$ Downing, 'SL Smyth*, ${ }^{2} \mathrm{~J}$ Thompson, ${ }^{1} \mathrm{M}$ Alazzam. ${ }^{1}$ Churchill Hospital, Gynaecological Oncology, Oxford, UK; ${ }^{2}$ John Radcliffe Hospital, Anaesthetics, UK

\subsection{6/ijgc-2021-ESG0.286}

Introduction/Background* Significant intraoperative blood loss can be encountered in complex pelvic surgery and impact on post-operative outcomes. There are a number of key anaesthetic techniques that can be used to optimise a patient in preparation for and to mitigate against predicted and unpredicted blood loss.

Methodology We performed a systematic review of the literature to identify the current perioperative anaesthetic strategies for optimisation of the patient and reducing blood loss in complex pelvic surgery.

Result(s)* This article explores the pre-operative and intraoperative techniques to reduce both the volume and physiological impact of blood loss.

Preoperative optimisation aims to identify patients that are at risk. This includes those with significant comorbidities or abnormal clotting disorders. Anticoagulation/antiplatelet therapies may require adjustment. Pre-operative anaemia should also be managed.

Intraoperative techniques are essential for reducing the rate of blood loss. Hypothermia leads to reversible disruption of the coagulation cascade and therefore techniques to maintain normothermia are required. Relative hypotension and avoidance of tachycardia both reduce blood loss but require close monitoring to ensure adequate tissue perfusion. Using regional anaesthesia can further support this. The use of pharmacological agents, such as tranexamic acid, have been demonstrated to reduce blood loss in pelvic surgery. Blood products such as clotting factors can specifically target and correct coagulopathies using point of care testing. Cell salvage allows use of autologous blood and clotting factors, reducing the need for cross-matched blood and the associated risks of transfusion.

Notably, reducing blood loss into the surgical field allows for improved visualisation of the anatomy and performance of surgical instruments used to cauterise bleeding, thus further improving haemorrhage control.

Conclusion* In conclusion, minimising blood loss during surgery requires input from the multidisciplinary team. Preoperative assessments should be held in a timely fashion to implement strategies for patient optimisation. Careful consideration and planning should be given to the intraoperative stage. Application of the strategies mentioned in this review will reduce the risk of significant intraoperative haemorrhage, aiming to improve the morbidity and mortality associated with complex pelvic surgery.

\section{LAPAROSCOPIC REPAIR OF EXTERNAL ILIAC VEIN INJURY}

${ }^{1} \mathrm{C}$ Yalcinkaya*, ${ }^{2} \mathrm{Gl}$ Aytok. 'Umraniye Research Hospital, Gynaecological Oncology, Istanbul, Turkey; ${ }^{2}$ Umraniye Research Hospital, ObstetricsandGynaecology, Istanbul, Turkey

\subsection{6/ijgc-2021-ESGO.287}

Introduction/Background* Endometrial Cancer is the most common gynecological cancer in developed countries. Hysterectomy is the main treatment and minimally invasive surgery is the preferred approach. Pelvic and paraaortic lymphadenectomy should be considered according to the histological type, grade and stage.

Methodology We describe a case of a 48-year old woman with endometrial cancer where an external iliac vein injury occured during the pelvic lymphadenectomy.

Result(s)* We succesfuly managed this injury with intracorporal suturing by using bulldog clamps and polypropylene sutures.

Conclusion* The management of a vascular injury during laparoscopy depends on its severity, location.In our case, external iliac vein injury occurred during the pelvic lymphadenectomy, which was managed effectively and safely.

\section{MINIMALLY-INVASIVE PELVIC EXENTERATION: A SURVIVAL ANALYSIS}

${ }^{1} \mathrm{~N}$ Bizzarri ${ }^{*},{ }^{2} \mathrm{~V}$ Chiantera, ${ }^{1} \mathrm{~S}$ Gueli Alletti, ${ }^{1} \mathrm{M}$ Loverro, ${ }^{1} \mathrm{C}$ Conte, ${ }^{1} \mathrm{E}$ Perrone, ${ }^{3} \mathrm{~A}$ Ercoli, ${ }^{1} \mathrm{~V}$ Gallotta, ${ }^{1} \mathrm{~L}$ Tortorella, ${ }^{1} \mathrm{~A}$ Fagotti, ${ }^{1} \mathrm{~F}$ Fanfani, ${ }^{1} \mathrm{G}$ Scambia, ${ }^{1} \mathrm{G}$ Vizzielli. ${ }^{1}$ Fondazione Policlinico Universitario A. Gemelli IRCCS, UOC Ginecologia Oncologica- Dipartimento per la salute della Donna e del Bambino e della Salute Pubblica, Rome, Italy; ${ }^{2}$ University of Palermo, Department of Gynecologic Oncology, Rome, Italy; ${ }^{3}$ Policlinico G. Martino, PID Ginecologia Oncologica e Chirurgia Ginecologica Miniinvasiva, Messina, Italy

\subsection{6/ijgc-2021-ESGO.288}

Minimally-invasive pelvic exenteration: a survival analysis. Introduction/Background* Pelvic exenteration for recurrent and persistent gynecological malignancies is traditionally performed with open approach (OA). Nevertheless, reports on the use of minimally-invasive surgical (MIS) approach to pelvic exenteration have been published with promising results in terms of peri-operative morbidity. However, oncological safety of this approach has been poorly investigated. The aim of the present study was to assess the disease-free survival (DFS) and overall survival (OS) of patients undergoing minimally-invasive pelvic exenteration.

Methodology All patients undergoing pelvic exenteration for gynecological cancers between 2010 and 2021 were included and divided into minimally invasive and open pelvic exenterations. Only patients who underwent OA with maximum tumor diameter $\leq 50 \mathrm{~mm}$ were included in order to balance characteristics of the two groups. Survival analysis was performed according to Kaplan Meier methods and log-rank test; multivariate analysis was performed with Cox regression.

Result(s)* Eighty-three patients were included. 35 (42.2\%) were in the MIS and $48(57.8 \%)$ in the OA group. 21 $(60.0 \%)$ and 14 (40.0\%) MIS were laparoscopic and robotic, respectively. Characteristics of the two groups are reported in table 1. Patients undergoing OA experienced a higher rate of 30-day post-operative complications $\geq$ grade 3 (table 2). With a median follow up of 12 months (range, 1-97), the median DFS was 11 months (95\%CI 8.8-13.2) versus 13 months 